\title{
AN INVESTIGATION ON THE LOGICAL STRUCTURE OF MATHEMATICS (VII) ${ }^{1)}$
}

\author{
SET-THEORETICAL CONTRADICTIONS
}

\author{
SIGEKATU KURODA
}

\section{Preliminaries}

For an investigation on the foundations of mathematics to get an adequate mutual understanding, it is necessary to describe the generalities of the investigation with the parallel description of its particularities. Although one should obtain an exact and precise knowledge only through the latter, it is almost impossible, without the former, to get the underlying ideas and the fundamental principles, upon which the investigation is based.

A logical system was defined in Part (I) and, after studying the fundamental properties of the logical system, it was denoted by ${ }^{2)}$ UL (Universal Logic). Although some generalities were described in the introductions in Parts (I) and (II) it seems necessary to describe some information about the position of the investigation, specifically about the conception of the nature of settheoretical parodoxes and about the way of formulating special branches of mathematics in UL. For, the position of the investigation has mainly developed by the consideration of these two subjects. In the following lines, therefore, I shall describe the generalities of UL in limiting the description to these two subjects.

Before doing this, it is to be remarked that the way of expression may be common to all such descriptions of generalities. Namely, we should use in them some words rather vaguely, relying on the traditional usage, association, and imagination, which the words are carrying; and other words in the precise sense of which the definitions are, however, given in detail in the description of the particularities of the investigation. Therefore, some inexactness or imperfection, however regrettable it may be, seems to be unavoidable, where the quick

Received July 9, 1958.

1) This is the Part (VII) of my papers with the same major title. The knowledge of Part (I) and (II) (Hamburger Abhandlungen and of Part (IV) (Nagoya Math. J. vol. 13 (1958)) are assumed.

2) Cf. the beginning of the introduction in Part (II). 
mutual understanding on the leading ideas of the investigation is mainly aimed at. Therefore, the ultimate exact information must, after all, be obtained by the description of particularities in detail. Even though, a description of generalities is useful and, in some cases, indispensable, so far as the transmission of our thought should be accomplished by an unspecified language common to us all.

\section{Set-theoretical "paradoxes"}

Semantical paradoxes, like Richard's, are not considered here, but exclusively syntactical contradictions, like Russell's, Cantor's, Burali-forti's and so on, so that the contradictions are to be considered to occur with respect to some specified logical system or to some specified language, here to UL.

As an example, we shall consider the typical syntactical contradiction, i.e., Russell's. Let, namely, a set $\mathrm{R}$ be defined by the defining formula

$$
\forall u . u \in \mathrm{R} \equiv u \notin u \text {. }
$$

Assuming that the quantifying range of $\forall u$ in (1) contains $\mathrm{R}$ itself, we get straightforwardly from (1) the contradiction

$$
\mathrm{R} \in \mathrm{R} \equiv \mathrm{R} \notin \mathrm{R}
$$

by substituting $\mathrm{R}$ for $u$ in (1). There have been many discussions about Russell's "paradox"; but where is an illegitimate reasoning in such simple logical inferences, each step of which is quite usual in logic? Our assumptions are two: the assumption of the existence of $R$ by the defining formula (1) and the assumption concerning the quantifying range of the element variable $u$ of R. It is evident that we get legitimately the contradiction (2) under these two assumptions.

Indeed, impredicative reasoning such as found in an inference of the deduction of Russell's contradiction is a particular reasoning, well distinguished from the predicative one. However, instead of rejecting impredicative inferences occurring in the above deduction, together with all those occurring in mathematics, we shall find later that the cause of the contradictions lies in some other respects.

\section{Contradictions in logic}

Logic develops where there is a contradiction. Before the set-theoretical contradictions entered into the field of logic, the syntactical contradictions in 
logic had been all sentential ones, as are found in predicate logic. Any one of these contradictions is deduced legitimately from a finite number of assumptions. We have to accept the simple fact that a certain contradiction is deduced legitimately under certain assumptions. This is quite of the same nature as in the case of set-theoretical contradictions. However, in the case of a sentential contradiction we can always deny any one of the assumptions which lead to the contradiction. This is a very simple way to avoid the contradiction so as to keep the law of contradiction valid. As for the set-theoretical contradictions, there is no such simple way to control these contradictions. This is a completely different nature compared with the case of sentential contradictions. However, it is a usual matter in logic that a contradiction is legitimately deduced from some assumptions.

\section{Assumptions of set-theoretical contradictions}

Now, we examine the assumptions of set-theoretical contradictions in order to examine whether or not there is something to be denied. As is already revealed in Russell's typical example, the essential part of the assumptions of set-theoretical contradictions consists generally in the range of set-variables and in the defining formulas of some finite number of sets, eventually including the principle of extensionality.

We consider the former first. The variable in logic is one of the primitive, undefinable notions. For instance, the variable for individuals in predicate logic is the variable for individuals; the variable for formulas is the variable for formulas: nothing else. No particular properties are to be attributed to variables in logic other than the rules of use of the variables in the logical system to be defined. Indeed, there may be a finite or infinite number of types of variables in a logical system, for instance, as variables for individuals or for formulas in predicate logic.

When we treat set variables, there are ways to classify the types of variables, for instance, into two kinds: variables for sets and those for classes. There may be infinitely many ways to define logical systems with set variables by introducing finite or infinite number of types of variables. It is also true that, if the set variables are not classified into, at least, two types, then the logical system may turn out to contain infinitely many number of contradictions. However, the cause of set-theoretical contradictions might not necessarily lie 
in the confusion of types of variables but in a finer nature of logical inferences, as will be shown later by taking the natural-number theory as an example.

Moreover, sets, classes, concepts, functions, mappings, etc. are considered to be the formal logical notions which belong ultimately to the same category, and the notion of types of variables is a notion corresponding to categories. Therefore, there might be no sufficient reason to classify the types of variables at the very starting point of the research, and so, the logical system UL with universal set-variables as unique type of variables was formulated, naturally under the sacrifice of an infinitely many number of contradictions entering into UL. However, it would be better to embrace contradictions within the logical system in order to control them.

As a consequence, the universal constant $\mathrm{V}$ with the characteristic property

$$
\forall x . x \in \mathrm{V}
$$

has any "set" (dependent variable) definable in UL as its element," and the null 0 with the characteristic property

$$
\forall x . x \notin 0
$$

has the dual property that no dependent variable definable in UL can be an element of 0 . Thus, the quantifier in UL and that in the intuitive logic acquire some analogy. For the latter which ranges, for instance, over real numbers any real number constructed intuitively can be an instance for the bound variable, while for the former any object defined formally in UL can be an instance for it.

Next, we consider the defining formulas of dependent variables (sets and concepts). Since the trend of the investigation aims at the general character as far as possible, no restriction should be laid also in defining sets and concepts. Therefore, a dependent variable $p=p^{x_{1}, \ldots, x_{n}}$ is introduced recursively into UL by a defining formula

$$
\forall u . u \in p^{x_{1}, \ldots, x_{n}} \equiv F^{u ; x_{1}, \ldots, x_{n}}
$$

where $F$ is any arbitrary well formed formula with $\in$ as unique dyadic relation and with $u, x_{1}, \ldots, x_{n}$ as complete system of free variables in $F$. In $F$ may occur any dependent variables previously defined."

3) Cf. \& 10 for the universal constant V.

4) Cf. the introduction in Part (I) and also $\$ 5$, Part (I). 
In this way, owing to such general character of the attitude of the investigation, it turns out that there is no way to deny any one of the assumptions from which set-theoretical contradictions are deduced. This is a remarkable nature which distinguishes the set-theoretical contradictions from the sentential.

\section{Proof in UL}

To observe such a general logical system as UL, there must be some restriction. I shall next explain this. Any variable in UL is either an independent variable such as $x, y, \ldots, u, \ldots$, or a dependent variable, including constant, such as $0, \mathrm{~V}, \mathrm{R}, p^{\mathrm{r}_{1}, \ldots, x_{n}}, \ldots$, defined by some defining formulas.

Now, in a proof, we use usually following inferences when we substitute a dependent variable $p$ for a bound variable $x$ :

(i) $\forall x F^{x}$ has been proved. Therefore, we have $F^{p}$.

(ii) In order to prove $>\forall x F^{x}$ we shall prove $>F^{p}$.

(iii) $7 \exists x F^{x}$ has been proved. Therefore, we have $7 F^{p}$.

(iv) In order to prove $\exists x F^{x}$ we shall prove $F^{p}$.

In this way, a finite number of dependent variables, like $p$ above, are used in a proof $P$ for a formula, say $H$. The formula of the principle of extensionality :

$$
\forall x y z . x=y \wedge x \in z \rightarrow y \in z
$$

may be also used as premise in $P$ (cf. $\S 11$, Part (I)). All the dependent variables, used in $P$, together with those which are needed to define them, should be collected. Let $\sigma$ be the sequence of the defining formulas of the collected variables, eventually including the formula (I). We call $\sigma$ the premises of the conclusion $H$ or of the proof $P$, and $P$ the proof for the assertion $\sigma+H$. Thus, a proof with $H$ as conclusion, or more generally the provability of $H$, is defined only when the premises $\sigma$ are explicitly given. This is a strong restriction for the concept of UL-proof, although it restricts in no way the system UL itself. In this way, what we can call proof in mathematics can be a proof in UL.

However, if the premise $\sigma$ contains, for instance, Russell's $\mathrm{R}$, then $\sigma$ t $H$ has a proof for any $H$, since a contradiction implies every formula. However, if we restrict the proof in UL to irreducible proof,,$^{5)}$ then it holds that a contradiction

5) Cf. Chapter V, Part (II) and also Introduction in Part (I). 
does not imply every formula irreducibly, and everything can be put in order. This is a weak but important way to control the set-theoretical contradictions: in the irreducible theory the law of contradiction does not hold in the sense that both $\sigma t H$ and $\sigma t>H$ may be irreducibly proved for some $H$ and $\sigma^{5}{ }^{5}$ I do not enter here into details of irreducible theory, but would like only to mention that the proof for Russell's contradiction in $\S 2$ is legitimate since it is irreducible.

Our main concern here is the bearing of set-theoretical contradictions to consistent theories in UL.

\section{Consistent theory and set-theoretical contradiction}

Let $T$ be a theory in UL and $\Sigma$ the species of all sets of $T$. A set in a proof is, roughly speaking, a dependent vairable which is substituted for a bound variable, at least once at a position in the proof in the inferences given above in $\S 5$, in such a manner that the dependent variable can not be eliminable from the proof. The species $\Sigma$ of sets of the theory $T$ is the species of those and only those variables which are allowed to use as sets in any proof in $T^{6}{ }^{6}$

Now, if we metalogically know that no contradiction can be proved for any closed $^{7}$ sequence $\sigma$ of formulas, consisting of a finite number of defining formulas of variables belonging to $\Sigma$ and of the formula (I), then the theory $T$ is consistent (cf. Introduction in Part (I)).

Any dependent variable can be used as "concept" in any proof of $T$ without destroying the consistency of $T$. Also any dependent variable $p$ can be used even as set without destroying the consistency of $T$, provided that the defining formula of $p$ is only used to "understand" the meaning, or more precisely, that no proof constituent is associated in any proof in $T$ to the defining formula of $p$. For instance, the formulas $R \in V, R \notin 0$ etc., are theorems of any consistent theory, where $R$ is Russell's $R$. In this way, the universal constant $V$ contains any consistent and inconsistent "set" as its element in any consistent theory.

The use of inconsistent sets in a consistent theory, explained above, is rather a trivial example for the legitimate use of (inconsistent) dependent variables. In order to show an untrivial example of the legitimate and illegitimate use of a dependent variable and also to show a way to formulate a theory of mathematics in UL, the formulation of a natural-number theory in UL is treated in

6) Cf. Part (X), fortheoming.

i) Cf. \$11, Part (I). 
the next article.

\section{Formulation of a natural-number theory in UL}

As is stated in the introduction in Part ( $\mathrm{I}$ ) the set $\mathrm{N}$ of all natural numbers is defined by the defining formula

$$
\forall u: u \in \mathrm{N} \equiv \forall x: 0 \in x \wedge \forall y . y \in x \rightarrow y^{\prime} \in x . \rightarrow u \in x,
$$

where 0 is the null constant and the successor $y^{\prime}$ of $y$ is $\{y\}$, the definitions of which are as usual. The natural number theory $T_{1}(N)$ is defined and its consistency is proved in Part (VIII). ${ }^{8)}$

We shall prove in $T_{1}(N)$ the following formulas: ${ }^{()}$(premises are omitted)

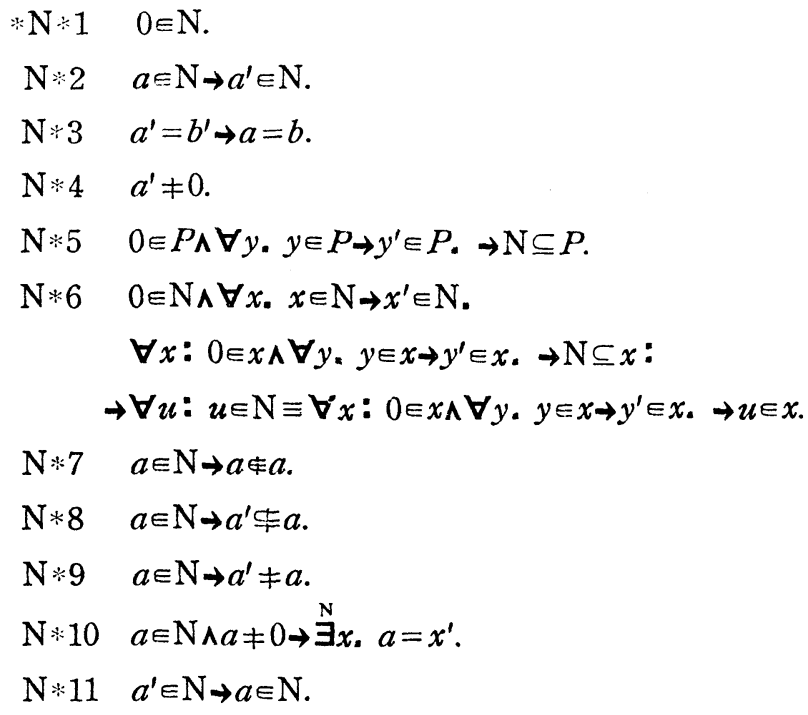

The dependent variables used as sets in the following proofs of the formulas $\mathrm{N} * 1-\mathrm{N} * 11$ are only as follows: ${ }^{10}$

8) Appearing in this same volume.

9) All formulas except $* \mathrm{~N} * 1$ are irreducible, namely the proofs given below are all irreducible except that for $* \mathrm{~N} * 1$. But the irreducibility is not our concern at present.

10) Particular attention is required when a formula $C\left(\equiv F_{p}^{a}\right)$ obtained from a formula $F^{a}$, previously proved, by replacing $a$ by a dependent variable $p$ is used as cut formula of an ordinary cut in a proof $P$. For, if the variable $a$ is used in the proof of $F^{* t}$ as a substitute for some bound variable, then $p$ is a set in the proof of $C\left(\equiv F_{b}^{a}\right)$, accordingly also in the proof $P$. If this is not the case, then $p$ is not necessarily a set in $P$, since the cut formula $C\left(\equiv F_{p}^{a}\right)$ is proved without using $p$ as set. For instance, the cut formula of the cut $\mathrm{N} * 10$ in the proof of $\mathrm{N} * 11$ is the formula obtained from $\mathrm{N} * 10$ by replacing $a$ by $a^{\prime}$ and $a$ is used in the proof of $\mathrm{N} * 10$ as set at the right above of the proof, since $a$ is substituted for the element variable $u$ of the set $P$ for induction. Therefore $a^{\prime}$ is a set in the proof of $\mathrm{N} * 11$.

The variable $P$ in $\mathrm{N} * 5$ is an independent variable. 
(i) The variable isological to $\{a\}$ in the proofs of $\mathrm{N} * 2, \mathrm{~N} * 7, \mathrm{~N} * 8, \mathrm{~N} * 9$, $\mathrm{N} * 10$, and $\mathrm{N} * 11$;

(ii) 0 in the proofs of $* \mathrm{~N} * 1, \mathrm{~N} * 7, \mathrm{~N} * 8, \mathrm{~N} * 9, \mathrm{~N} * 10$, and $\mathrm{N} * 11$;

(iii) $\mathrm{N}$ in the proof of $\mathrm{N} * 6$;

(iv) Russell's set $\mathrm{R}$ as set for induction in the proof of $\mathrm{N} * 7$, accordingly in those of $\mathrm{N} * 8$ and $\mathrm{N} * 9$;

(v) $\mathrm{P}$ in $\mathrm{N} * 10$, defined as set for induction, accordingly in $\mathrm{N} * 11$.

In the following proofs, the premises are omitted. $\ddot{\forall} x F^{x}$ and $\exists^{a} x F^{x}$ mean the restriction of the quantifying range of $x$ to $a$, namely $\forall x . x \in a \rightarrow F^{x}$ and $\exists x . x \in a \wedge F^{x}$, respectively.

$* \mathrm{~N} * 1 \quad 0 \in \mathrm{N}$

$$
\begin{aligned}
& \frac{-0 \in N}{(w)-\forall x: 0 \in x \wedge \forall y \cdot y \in x \rightarrow y^{\prime} \in x . \rightarrow 0 \in x} \\
& * \text { Spf. } \forall y . \quad y \in w \rightarrow y^{\prime} \in w
\end{aligned}
$$

$\mathrm{N} * 2 \quad a \in \mathrm{N} \rightarrow a^{\prime} \in \mathrm{N}$

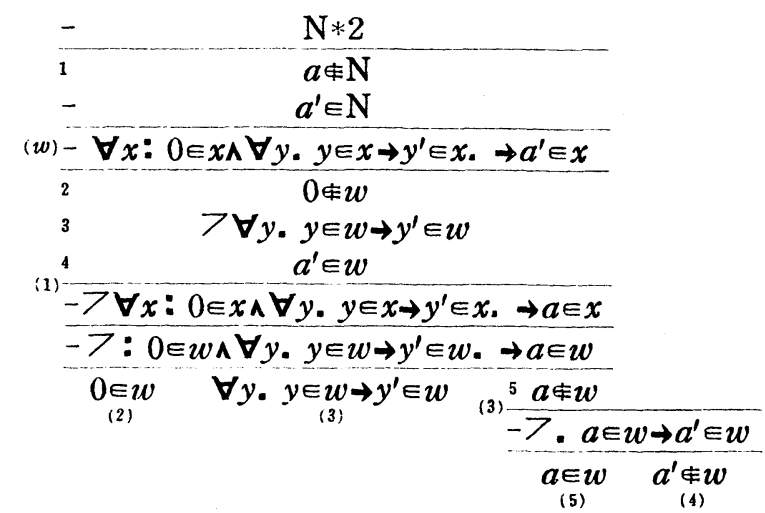

$\mathrm{N} * 3 \quad a^{\prime}=b^{\prime} \rightarrow a=b$

$\mathrm{N} * 4 \quad a^{\prime} \neq 0$
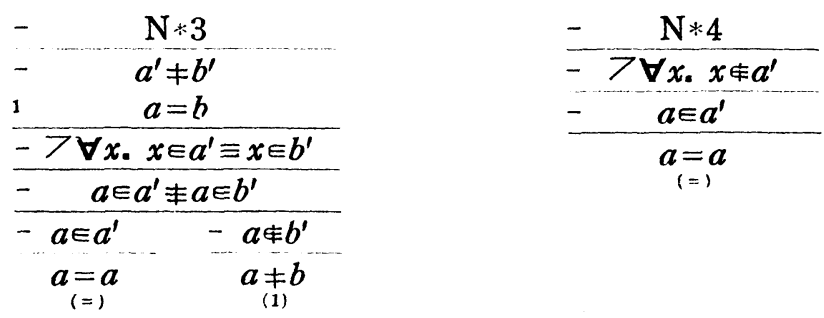

The converse $a=b \rightarrow a^{\prime}=b^{\prime}$ of $\mathrm{N} * 3$ is a speeial case of the metatheorem for equality. 
$\mathrm{N} * 5 \quad 0 \in P \wedge \forall y, y \in P \rightarrow y^{\prime} \in P . \stackrel{*}{\rightarrow} \subseteq P$

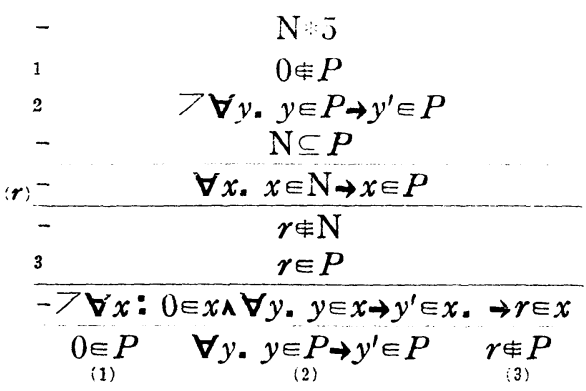

$\mathrm{N} \therefore 6 \quad 0 \in \mathrm{N} \wedge \forall x . x \in \mathrm{N} \rightarrow x^{\prime} \in \mathrm{N} . \wedge \forall x: 0 \in x \wedge \forall y . y \in x \rightarrow y^{\prime} \in x . \rightarrow \mathrm{N} \subseteq x:$

$\rightarrow \forall u: u \in \mathrm{N} \equiv \forall x: 0 \in x \wedge \forall y . y \in x \rightarrow y^{\prime} \in x . \rightarrow u \notin x$

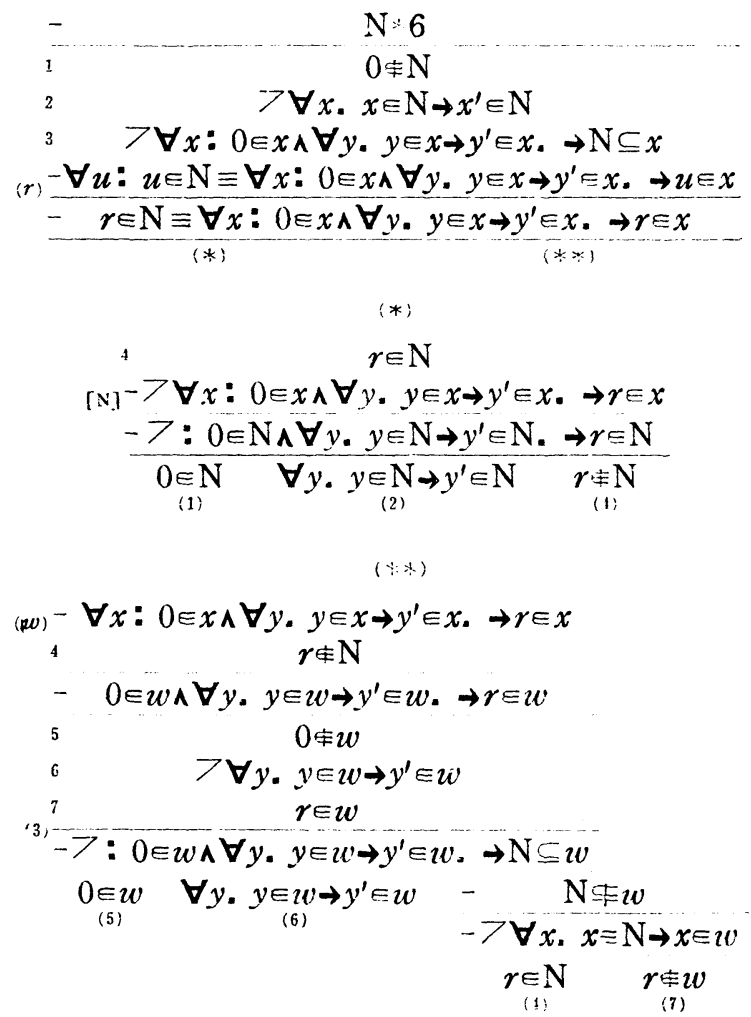

The formulas $\mathrm{N} * 1-\mathrm{N} * \overline{5}$ are Peano's system of axioms for the natural number theory. Therefore by $\mathrm{N} * 6$ we see that the set $\mathrm{N}$ characterizes the set of all natural numbers as in Peano's system of axioms. 
$\mathrm{N} * 7 \quad a \Subset \mathrm{N} \rightarrow a \notin a$

Define $\mathrm{R}$ by $u \in \mathrm{R} \equiv u$ 就.

$$
\begin{aligned}
& \begin{array}{ll}
- & \mathrm{N} * 7 \\
- & a \notin \mathrm{N}
\end{array} \\
& 1 \quad a \notin a \\
& {[x]->\forall x ; 0 \in x \rightarrow \forall y . y \in x \rightarrow y^{\prime} \in x . \rightarrow a \in x} \\
& -0 \in \mathrm{R} \quad(s)-\forall y, y \in \mathrm{R} \rightarrow y^{\prime} \in \mathrm{R} \quad-a \notin \mathrm{R} \\
& 0 \text { 我 } \\
& s \nsubseteq \mathrm{R} \\
& \begin{array}{lc}
- & s^{\prime} \in \mathrm{R} \\
\hline 2 & s \in S \\
- & \boldsymbol{s}^{\prime} \notin s^{\prime} \\
\hline- & \boldsymbol{s}^{\prime} \neq s
\end{array} \\
& a \in a \\
& 0 * 1 \\
& ->\forall x . x \in s^{\prime} \equiv x \in s \\
& \text { - } \quad s \in s^{\prime} \neq s \in S \\
& \underset{\substack{s=S \\
(=1}}{-s \in s^{\prime}} \quad \underset{(2)}{s \notin S}
\end{aligned}
$$

In this proof, Russell's set $R$ is used as set for induction. This is legitimate, because our reasoning remains within the consistent theory $T_{1}(N)$.

$\mathrm{N} * 8 \quad a \in \mathrm{N} \rightarrow a^{\prime} \nsubseteq a$

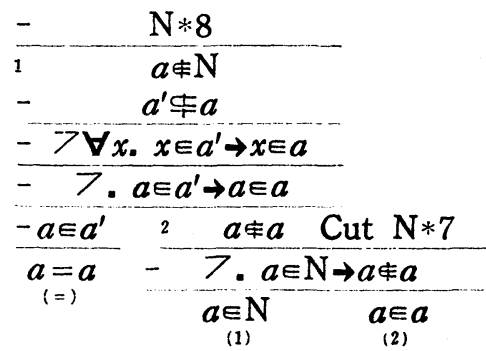

From $\mathrm{N} * 8$ follows

$\mathrm{N} * 9 \quad a \in \mathrm{N} \rightarrow a^{\prime} \neq a$

$\mathrm{N} * 10 \quad a \in \mathrm{N} \wedge a \neq 0 \rightarrow \mathrm{N} x . a=x^{\prime}$

Define the set $\mathrm{P}$ for induction by $u \in \mathrm{P} \equiv u=0 \mathrm{v} \stackrel{\mathrm{N}}{\exists} x \cdot u=x^{\prime}$.

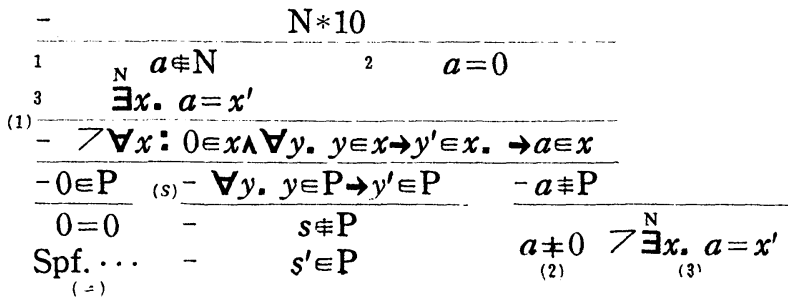




$$
\begin{aligned}
& 4 \text { ᄀ. } s=0 \text { v 当x. } s=x^{\prime} \\
& \text { - } s^{\prime}=0 \mathrm{v} \exists x . s^{\prime}=x^{\prime} \\
& \text { Spf. } \quad s^{\prime}=0 \\
& \text { - } \quad \mathrm{N} x, s^{\prime}=x^{\prime} \\
& \text { - } \quad s \in \mathrm{NA} s^{\prime}=s^{\prime} \\
& \text { (4) }
\end{aligned}
$$

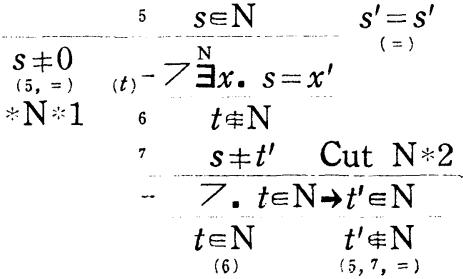

$\mathrm{N} * 11 \quad a^{\prime} \in \mathrm{N} \rightarrow a \in \mathrm{N}$

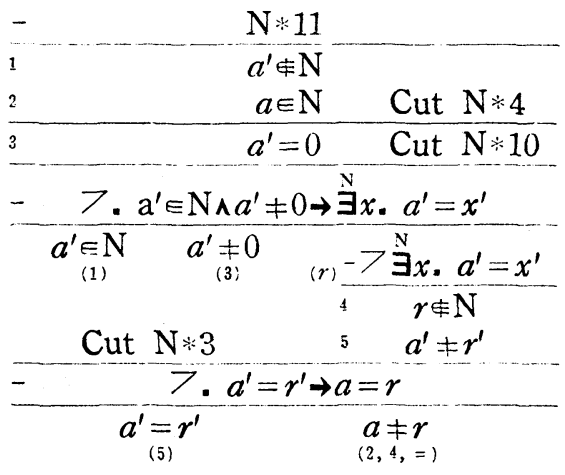

\section{Legitimate and illegitimate use of dependent variables}

As is seen in the proof of $\mathrm{N} * 7$, from which the formula $\mathrm{N} * 9 \quad a \in \mathrm{N} \rightarrow a^{\prime} \neq a$ follows, the legitimate use of Russell's set $R$ in the consistent theory $T_{1}(N)$ is allowed. From this fact we see that the cause of the set-theoretical contradictions consists neither in the assumption of the existence of a certain set nor in the fact that the quantifiers range over the domain which contains inconsistent sets but in the way of use of the inconsistent sets in a proof. Therefore, in order to find the cause of the set-theoretical contradictions more detailed analysis of logical inferences is required. The author can not find at present any general criteria for it: it seems there is none of such.

Recapitulating the nature of set-theoretical "paradoxes", we can say as follows. An illegimate use of a dependent variable is the use of the variable at a position in a proof of a consistent theory in such a way that the use of the variable at the position in the proof is not allowed by the definition of the 
theory. Any use of any dependent variable in an irreducible proof is legitimate. In this case any set-theoretical paradox gives rise to at least one irreducible proof of contradiction in UL.

\section{Formulation of axiomatic theories in UL}

We have deduced the system of Peano's axioms from the defining formula of N. As is stated in $\S 21$, Part (II), about the definition of $0, \mathrm{~V}$, and the ordered $n$-tuple $\left\langle m_{1}, \ldots, m_{k}\right\rangle$, there is arbitrariness also in the definition of N. Namely, we may use some other dependent variables than those we applied as the number 0 and the successor $y^{\prime}$ of $y$. In any case, once the system of Peano's axioms is proved, we can use Peano's axioms as premises in the natural number theory $T_{1}(N)$ instead of the defining formula of $N$. This is the way to formulate an axiomatic theory in UL. The important thing is that all the axioms of an axiomatic theory in UL must, at first, be deduced from the defining formulas of a finite number of dependent variables. ${ }^{11}$

As another example for an axiomatic theory in UL we shall treat one from among the branches of abstract mathematics to make contrast against natural number theory as concrete mathematics. Namely, we shall define the concept of topological space ${ }^{12)}$ in UL, i.e. a dependent variable $\mathrm{TpSp}^{\mathscr{D}}$, where $\mathscr{D}$ is an independent variable and $E \in \mathrm{TpSp}$ " is read " $E$ is a space on which a topological structure is defined by $\mathfrak{D}$ " or " $E$ is a topological space with $\mathfrak{D}$ as the set of all open sets of $E^{\prime \prime}$.

First, we define $\mathfrak{P}(a), \subseteq(a)$ and $a \cap b$, as usual, respectively by ${ }^{13 \prime}$

$$
\begin{aligned}
& u \in \mathfrak{P}(a) \equiv u \subseteq a, \\
& u \in \subseteq(a) \equiv \exists x . u \in x \wedge x \in a, \\
& u \in a \cap b \equiv u \in a \wedge u \in b .
\end{aligned}
$$

11) Let $T$ be an axiomatic theory in UL, which is assumed to be consistent and to contain the natural number theory to some sufficient extent. Then by Gödel's theorem there is a formula $A$ in $T$ which is unprovable in $T$ and intuitively holds. Then, as was stated by Gödel, we get a consistent axiomatic theory $T^{\prime}$ by adjoining the negation of $A$ to the system of axioms of $T$, and in $T^{\prime}$ some theorems, like $>A$, contradict our intuitive knowledge. However, $T^{\prime}$ is not an axiomatic theory in UL, unless $7 A$ has been proved in some consistent extension of $T$ in UL. It is, therefore, an open problem, whether there is a consistent axiomatic theory in UL, which includes the natural number theory to sufficient extent, and in which a provable formula contradicts our intuitive knowledge.

12) Cf. for instance, N. Bourbaki: Élements de mathématique, Première partie, Livre III. Chapitre I, Structures topologiques, Paris, 1951, pp. 9, 10.

13) The quantifier $\forall u$ for the element variable $u$ is omitted. 
Then, we define two constants $\mathrm{O}_{1}$ and $\mathrm{O}_{2}$ and a dependent variable $\mathrm{P}^{E}$ depending on an independent variable $E$, respectively, by

$$
\begin{aligned}
& u \in \mathrm{O}_{1} \stackrel{\stackrel{\Re(u)}{\forall} x .}{\subseteq}(x) \in u, \\
& u \in \mathrm{O}_{2} \equiv \stackrel{u}{\forall} x y . x \cap y \in u \text {, } \\
& u \in \mathrm{P}^{E} \equiv E \in u \text {. }
\end{aligned}
$$

Now, $\mathrm{TpSp}^{2}$ is defined by

$$
E \in \mathrm{TpSp}^{\complement} \equiv . \mathscr{D} \subseteq \mathfrak{B}(E) \wedge D \in \mathrm{O}_{1} \cap \mathrm{O}_{2} \cap \mathrm{P}^{E} .
$$

It is evident that if $E \in \mathrm{TpSp}$, then $E$ satisfies the axioms of topological space with $\mathcal{D}$ as the set of all open sets.

We do not enter here into the deduction of the theory of topological spaces. (To do this, we must, first of all, define the species of sets of the theory of topological spaces.) However, by these two examples, it has been shown how the axiomatic theories in mathematics can be formulated in UL. It is also seen that there is no difference in principle between the formulation in UL of abstract and concrete theories in mathematics. Specifically, the natural number theory is formulated in UI, in the generality of set theory. In this generality even the sets which give rise to "paradoxes" are legitimately used in the natural number theory.

\section{Universal constant $\mathrm{V}$}

Now, we observe the universal constant $\mathrm{V}$ which is defined by

$$
\forall u, u \in \mathrm{V} \equiv . u=u \text {. }
$$

Instead of the definiens $u=u$ we may use, to define $\mathrm{V}$, any formula $F^{u}$ in UL, which has only $u$ as free variable and is provable in UL without any premise; thereby bound variables but no dependent variables may occur in $F^{u}$.

The formula (3) is proved only by using the formula (7) as premise, and so, by (3),

$$
p \in \mathrm{V}
$$

is a theorem in UL for any variable $p .^{14)}$ Hence the universal constant $\mathrm{V}$ may be compared with Dedekind's "meine Gedankenwelt", ${ }^{5}$ ' although Dedekind did

14) In the proof of (8) the set $p$ is used only for "understanding".

15) Cf. Gesammelte mathematische Werke, Braunschweig, 1932, vol. III, p. 357. Cf. also Bolzano: Paradoxien des Unendlichen, Leibzig, $1851 \S 13$, which is mentioned by Dedekind, ibid. in the foot note. 
not have a precisely defined logical system in the sense of our age. Dedekind's expression "die Gesamtheit aller Dinge, welche Gegenstand meines Denkens sein können" restores a precise meaning in interpreting with respect to UL as "the constant V in UL that has as its element any dependent variable which can be defined in UL". The formula (8) is, therefore, not only a theorem in UL, but also a theorem of any consistent subsystem of $U L$. For instance, $V \in V, R \in V$, $\mathrm{N} \in \mathrm{V}$ etc. are theorems of the consistent natural number theory $\mathrm{T}_{1}(\mathrm{~N})$.

Generally, a theorem $\sigma \mathrm{H} H$ in UL is a theorem in any consistent subsystem of UL, provided that all the dependent variables used as sets in the proof of $\sigma$ t $H$ are sets of the subsystem. However, there are theorems of some consistent subsystems of UL, which are not theorems of other consistent subsystems of UL. Therefore, some properties of a dependent variable are naturally not invariant for all consistent subsystems of UL; therein a property of a dependent variable means a property of the variable which is expressed by a theorem or by a finite or infinite number of theorems of some particular consistent subsystems of UL. To this category belongs the problem whether $\mathrm{V}$ is finite or infinite.

We see in Part (VI) that

$$
\forall x, x=\mathrm{V}
$$

is a theorem in the consistent $\mathrm{V}$-system, so that $\mathrm{V}$ consists only of $\mathrm{V}$ itself, when $\mathrm{V}$ is considered in $\mathrm{V}$-system.

Let us denote by $T\left\{m_{1}, \ldots, m_{k}\right\}, m_{1}, \ldots, m_{k}$ being any dependent variables, the theory of which the species of sets consists of $m_{1}, \ldots, m_{k}$ and all elementary sets generated by $m_{1}, \ldots, m_{k}{ }^{161}$

The following formulas $\mathrm{V} * 0-\mathrm{V} * 4$ and $0 * 1-0 * 4$ are not only theorems of UL but also of the consistent subsystem $T\{0, \mathrm{~V}\}$ of $\mathrm{UL}$ : the sets used in the proofs are : $0, \mathrm{~V}$ in $\mathrm{V} * 0$ and $\mathrm{V} * 2 ; 0, \mathrm{~V},\{\mathrm{~V}\rangle$ in $\mathrm{V} * 3 ; 0, \mathrm{~V}, \mathrm{~V}^{\prime}, \ldots, \mathrm{V}^{(n-1)}$ in $\mathrm{V} * 4$.

$$
\begin{array}{ll}
\mathrm{V} * 1 & a \in \mathrm{V} \\
0 * 1 & a \notin 0 \\
\mathrm{~V} * 0 & 0 \neq \mathrm{V}
\end{array}
$$

$(0 \neq \mathrm{V}$ is expressed by $7 \forall x . x \notin \mathrm{V}$ or $7 \forall x . x \in 0$.

\footnotetext{
16) Cf. $\S 3$, Part (VIII), appearing in this same volume.
} 


$$
\begin{array}{cc}
\frac{-\mathrm{V} * 0}{-7 \forall x \cdot x \notin \mathrm{V}} & \frac{-7 \forall x \cdot x \in 0}{\mathrm{~V} \in \mathrm{V}} \\
\mathrm{V} * 1 & 0 \notin 0 \\
& 0 * 1
\end{array}
$$

$\mathrm{V} * 2 \quad \mathrm{~V} \neq\{a\}$

$0 * 2 \quad 0 \neq\{a\}$

$$
\begin{aligned}
& -\quad \mathrm{V} * 2 \\
& \begin{array}{c}
{[\mathrm{v}, 0]^{-} \quad 7 \forall x \cdot x \in\{a\}} \\
-\quad \mathrm{V \notin \{ a \}}
\end{array} \\
& \frac{-\quad 0 \notin\{a\}}{1} \\
& 0 \neq a \text { Cut }(=) \\
& -7.0=a \wedge a=\mathrm{V} \rightarrow 0=\mathrm{V} \\
& 0=a \quad a=\mathrm{V} \quad 0 \neq \mathrm{V} \\
& \text { (2) } \underset{\operatorname{Cut}(1)}{(1)}(\mathrm{V} * 0
\end{aligned}
$$

$\frac{\frac{-0 * 2}{-7 \forall x . x \notin\{a\}}}{-\frac{a \in\{a\}}{a=a}}$

$\mathrm{V} * 3 \quad \mathrm{~V} \neq\{a, b\}$

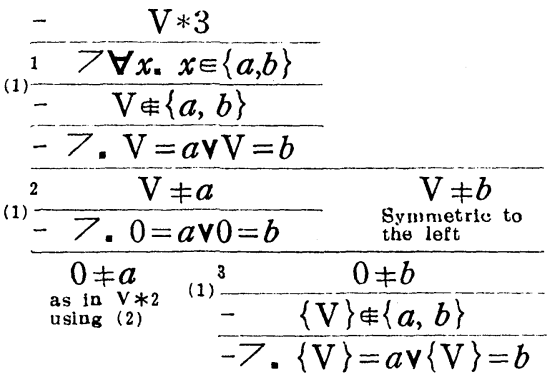

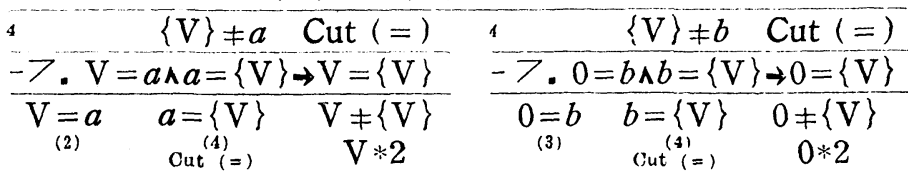

Now, denoting $\{m\}$ by $m^{\prime}$, we see that

$$
0,0^{\prime}, 0^{\prime \prime}, \ldots
$$

are all different each other: $0 \neq 0^{(n)}$ for $n \geq 1$ by $0 * 2$, and assuming $0^{\prime}, \ldots, 0^{(n}$ are different each other we have $0^{(r)} \neq 0^{(n+1)}$ for $r=1, \ldots, n$ by the formula $\mathrm{El} * 3^{17)}$ of elementary sets and by our assumption. Therefore,

$$
0^{(m)} \neq 0^{(n)} \quad\left(m \neq n, 0^{(0)}=0\right)
$$

are theorems for any metalogical number $m, n$ in any consistent subsystem of

17) Cf. Part (III), Nagoya Math. J. vol. 13 (1958). 
UL of which the species of sets contains any member of (10). Therefore, the infinite number of formulas (11) expresses the fact that $\mathrm{V}$ is infinite when $\mathrm{V}$ is considered in such a consistent subsystem of UL.

On the contrary, in the consistent subsystem $T\{\mathrm{~V}\}$ of $\mathrm{UL}$, it is unprovable even that there exist two different sets: $T\{\mathrm{~V}\}$ is a subsystem of the consistent $\mathrm{V}$-system and in $\mathrm{V}$-system all dependent variables are equal to $\mathrm{V}$; hence, the existence of two different sets in $T\{\mathrm{~V}\}$ contradicts the consistency of the $\mathrm{V}$-system. On the other hand, it is not difficult to prove that the formula (9) is unprovable in $T\{\mathrm{~V}\}$. Therefore we have

THEOREM. In the consistent subsystem $T\{\mathrm{~V}\}$ of $\mathrm{UL}$, it is undecidable whether there exist two different sets.

However, if the species of sets of a consistent subsystem $T$ of UL contains

$$
0, \mathrm{~V}, \mathrm{~V}^{\prime}, \mathrm{V}^{\prime \prime}, \ldots
$$

then the universal constant $\mathrm{V}$ considered in $T$ contains infinite sets. For, the proof of $\mathrm{V} * 2$ is a proof in $T$, and accordingly, $\mathrm{V} \neq\{\mathrm{V}\}$ is a theorem of $T$; hence, we can prove, recursively as before, that the sequence (12) consists of different sets each other ( $\mathrm{V} * 0$ and $0 * 2$ are used in the proof).

In such a theory $T$ we have as $T$-theorem

$$
\mathrm{V} \neq\left\{a_{1}, \ldots, a_{n}\right\}
$$

for any metalogical number $n$. Namely, $\mathrm{V} * 2$ is proved by using two different sets 0 and $\mathrm{V} ; \mathrm{V} * 3$ by three different sets $0, \mathrm{~V}$, and $\{\mathrm{V}\}$; and similarly $\mathrm{V} * 4$ is proved by using $n+1$ different sets $0, \mathrm{~V}, \mathrm{~V}^{\prime}, \ldots, \mathrm{V}^{(n-1)}$. The dual formula

$* 0 * 4$

$$
0 \neq\left\{a_{1}, \ldots, a_{n}\right\}
$$

is also a theorem (not irreducible) of $T$ (cf. the proof of $0 * 2$ ).

In this way, we have seen that there are consistent subsystems of UL, in which either $\mathrm{V}$ is proved to be finite, or infinite, or else it is undecidable whether $\mathrm{V}$ is finite or infinite.

What we have seen from the above description is that the universal constant $\mathrm{V}$, which corresponds to the logical universe of Bolzano-Dedekind can be used in UL with the same extent of rigorousness as we use the null constant 0 in UL. So we shall deduce the formulas $\mathrm{V} * 5-\mathrm{V} * 9$ concerning $\mathrm{V}$, which are theorems 
of $T\{\mathrm{~V}\}$. The only dependent variable used as set in the following proofs is $\mathrm{V}$ in the proof of $\mathrm{V} * 8$. Notice that $\mathrm{V}$ in other places as well as $\mathfrak{P}(a), \mathfrak{P}(\mathrm{V})$ and $\varsigma(a)$ are used as concepts.

$\mathrm{V} * 5$

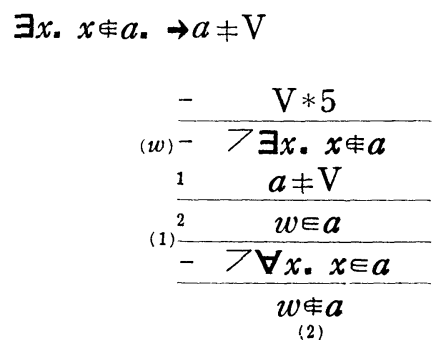

$$
* \mathrm{~V} * 6 \quad a \subseteq \mathrm{V}
$$

$$
\begin{aligned}
& \frac{\mathrm{V} * 6}{(w)-\forall x . \quad x \in a \rightarrow x \in \mathrm{V}} \\
& \begin{array}{l}
-w \in a \rightarrow w \in \mathrm{V} \\
* \mathrm{Spf} . \quad w \notin a
\end{array} \\
& w \in \mathrm{V} \\
& \mathrm{V} * 1
\end{aligned}
$$

$* \mathrm{~V} * 7 \quad \mathfrak{P}(\mathrm{V})=\mathrm{V}$

$$
\begin{gathered}
-\quad \mathrm{V} * 7 \\
(w)^{-} \forall x \cdot x \in \mathfrak{P}(\mathrm{V}) \\
-w \in \mathfrak{P}(\mathrm{V}) \\
w \subseteq \mathrm{V} \\
* \mathrm{~V} * 6
\end{gathered}
$$

$\mathrm{V} * 8 \quad \mathfrak{B}(a)=\mathrm{V} \rightarrow a=\mathrm{V}$

$\mathrm{V} * 9 \quad \exists x \stackrel{a}{\forall} y, x \notin y \rightarrow \Xi(a) \neq \mathrm{V}$

$$
\begin{array}{cc} 
& \mathrm{V} * 8 \\
- & \mathfrak{P}(a) \neq \mathrm{V} \\
-\quad a=\mathrm{V} \\
1 \quad 7 \forall x \cdot x \in \mathfrak{P}(a) \\
(w)^{-} \quad \forall x \cdot x \in a \\
{ }^{2} \quad \mathrm{w \in a} \\
-\quad \mathrm{V \notin P(a)} \\
-\quad \mathrm{V} \neq a \\
\hline-7 \forall x \cdot x \in \mathrm{V} \rightarrow x \in a \\
-\quad 7 \cdot w \in \mathrm{V} \rightarrow w \in a \\
w \in \mathrm{V} \quad w \notin a \\
\mathrm{~V} * 1 \quad \text { (2) }
\end{array}
$$$$
\begin{array}{cc}
- & \mathrm{V} * 9 \\
& a \\
(r)- & >\exists x \underset{\forall}{\forall} y \cdot x \notin y \\
1 & \Xi(a) \neq \mathrm{V}
\end{array}
$$$$
\text { (1) }{ }^{2} \quad>\ddot{\forall} y, r \notin y
$$$$
\text { (1) } 7 \forall x, x \in \Xi(a)
$$$$
\text { - } r \notin \subseteq(a)
$$$$
\text { (s) }->\exists x . x \in a \wedge r \in x
$$$$
\text { - 7. } s \in a \wedge r \in s
$$$$
s \notin a
$$$$
\text { (2) } \quad r \notin S
$$$$
\text { (2) }->. s \in a \rightarrow r \notin s
$$$$
\underset{(4)}{S \in a} \quad r \in S
$$

\section{Relation between $\mathrm{N}$ and $\mathrm{V}$}

Now, we observe the relation between $\mathrm{V}$ and $\mathrm{N}$. We shall first prove $\mathrm{N} * \mathrm{~N} \quad \mathrm{~N} \notin \mathrm{N}$

Define the set $\mathrm{P}$ for induction by $u \in \mathrm{P} \equiv u \neq \mathrm{N}$. 


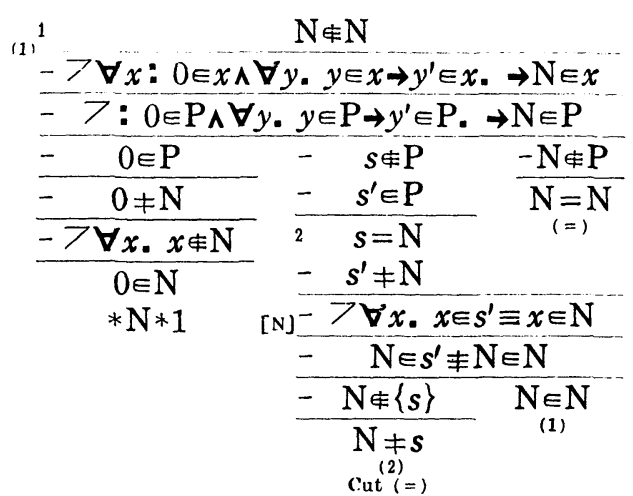

The constant $\mathrm{N}$ is used as set in this proof. The formula $\mathrm{N} * \mathrm{~N}$ is a theorem of the consistent theory $T_{1}(N)$. Since $T_{1}(N)$ is a consistent extension of $T\{0, V\}$, all the formulas deduced in $\S 10$ and $\S 11$ are theorems of $T_{1}(N)$. We have from $\mathrm{N} * \mathrm{~N}$ and $\mathrm{V} * 5$

$\mathrm{N} * 12 \quad \mathrm{~N} \neq \mathrm{V}$.

The formula $\mathrm{N} \in \mathrm{V}$ is a consequence from $\mathrm{V} * 1$. On the contrary we have $\mathrm{N} * 13 \quad \mathrm{~V} \notin \mathrm{N}$

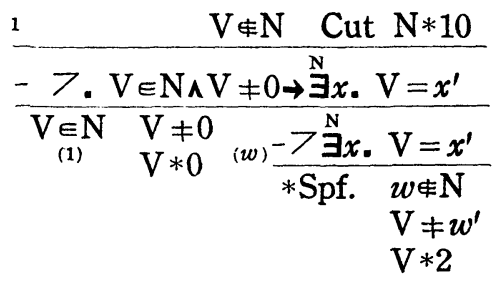

We define $\mathfrak{P}^{(n)}(a), \quad n=0,1,2, \ldots$, successively, by $\mathfrak{P}^{(0)}(a)=a, \quad \mathfrak{P}^{(n)}(a)$ $=\mathfrak{P}\left(\mathfrak{H}^{(n-1)}(a)\right)$. Then, we have from $\mathrm{N} * 12$ and $\mathrm{V} * 8$

$\mathrm{N} * \mathfrak{P}$

$$
\mathfrak{P}^{(n)}(\mathrm{N}) \neq \mathrm{V}
$$

for any metalogical number $n . \quad \mathrm{N} * \mathfrak{P}$ is also a theorem of the consistent natural number theory $T_{1}(N)$, a fortiori in any consistent extension of $T_{1}(N)$. Therefore, if $T_{1}(\mathrm{~N})$ can be extended to a certain consistent theory $T$ of finite types of variables with natural numbers as basic type, then $\mathrm{N} * \mathfrak{\beta}$ is also a theorem in $x$.

\section{Defining formula of $\mathrm{V}$}

We can define $\mathrm{V}$ by

$$
\forall u . u \in \mathrm{V} \equiv u \in u \mathrm{~V} u \notin u
$$


as is said in $\$ 10$. But no dependent variable may occur in the definiens of $V$. In order to show an example in which $\mathrm{V}$ is defined by other dependent variables, we shall use in this article the following definition of $\mathrm{V}$ :

$$
\begin{aligned}
& \forall u . u \in \mathrm{S} \equiv u \in u, \\
& \forall u . u \in \mathrm{R} \equiv u \notin \mathrm{S}, \\
& \forall u . u \in \mathrm{V} \equiv u \in \mathrm{S} \vee u \in \mathrm{R} .
\end{aligned}
$$

The closed sequence V, R, S, contains now Russell's set R. Russell's set R by this definition leads to contradiction. So is also $\mathrm{V}$. While $a \in \mathrm{V}$ is proved irreducibly as follows

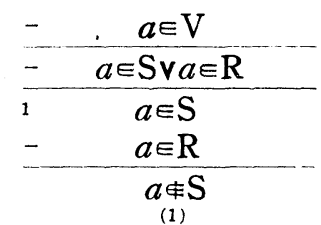

so that $\mathrm{R} \in \mathrm{V}$ is an irreducible truth, we have also $\mathrm{R} \notin \mathrm{V}$ irreducibly as follows:

$$
\begin{aligned}
& \text { - } \quad \frac{R \notin V}{R \in S V R \in R} \\
& \text { - } \mathrm{R} \notin \mathrm{S} \quad{ }^{1} \quad \mathrm{R} \notin \mathrm{R} \\
& 1 \mathrm{R} \notin \mathrm{R}-\mathrm{R} \in \mathrm{S} \\
& \text { (1) }-\mathrm{R} \in \mathrm{S} \quad \mathrm{R} \in \mathrm{R} \\
& \mathrm{R} \in \mathrm{R} \\
& \text { (1) }
\end{aligned}
$$

Moreover, we have the irreducibly proved formulas: $R \in S, R \notin S ; R \in R, R \notin R$. In each proof of these formulas as well as $R \notin V$, the constant $R$ is used as set, while in the above proof (14) of $a \in \mathrm{V}$, the constants $\mathrm{S}$ and $\mathrm{R}$ are used as concepts. Therefore $a \in \mathrm{V}$ with the definition (13) of $\mathrm{V}$ is a theorem of UL.

\section{Mathematical Institute}

Nagoya University 